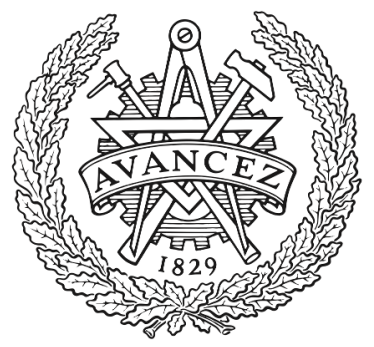

CHALMERS

UNIVERSITY OF TECHNOLOGY

\title{
An active load-pull technique creating time-variant impedances to emulate coupling between power amplifiers
}

Downloaded from: https://research.chalmers.se, 2023-04-26 15:35 UTC

Citation for the original published paper (version of record):

Nopchinda, D., Buisman, K. (2017). An active load-pull technique creating time-variant impedances to emulate coupling between power amplifiers. IEEE MTT-S International Microwave Symposium Digest: 1437-1440. http://dx.doi.org/10.1109/MWSYM.2017.8058889

N.B. When citing this work, cite the original published paper. 
This is the accepted version of a work that was accepted for publication in the following source: 2017 IEEE MTT-S International Microwave Symposium (IMS)

\title{
An active load-pull technique creating time-variant impedances to emulate coupling between power amplifiers
}

\author{
By D. Nopchinda and K. Buisman \\ This file was downloaded from: the Chalmers Research website \\ https://research.chalmers.se
}

Copyright (C) 2018, IEEE

Notice: Changes introduced as a result of the publishing process, such as editing and formatting may not be reflected in this document. For the final version of this work, please refer to the published source:

https://doi.org/10.1109/MWSYM.2017.8058889

N.B. When citing this work, cite the final version. 


\title{
An Active Load-Pull Technique creating Time-Variant Impedances to Emulate Coupling between Power Amplifiers
}

\author{
Dhecha Nopchinda and Koen Buisman \\ Chalmers University of Technology, SE-412 96 Gothenburg, Sweden
}

\begin{abstract}
A method for emulating antenna array coupling effects, based on active load-pull, to present time-varying impedances to power amplifiers is presented. An entire array, given identical elements, can be emulated using a single device-undertest. The method is demonstrated and verified by studying two scenarios, where the resulting adjacent channel power ratio and error-vector magnitude are given as function of delay and coupling for a $6 \mathrm{~W}$ GaN power amplifier. Differences in adjacent channel power ratio and error-vector magnitude can be attributed to timevariant load impedances.
\end{abstract}

Index Terms-5G mobile communication, arrays, power amplifiers, coupling, distortion, load-pull.

\section{INTRODUCTION}

Future communication systems, such as proposed for $5 \mathrm{G}$, will enable high bandwidth and low latency to users [1]. To reach this goal, a high number of active antennas at the basestation is envisioned. Keeping such a system compact and cost effective will require further integration of baseband, analog $\mathrm{RF}$, as well as antennas.

Such highly integrated, multi-antenna systems, will need to account for coupling effects, originating on-chip between interconnects as well as through antennas. In a conventional system, these effects have been diminished by the inclusion of isolators, however for integrated mm-wave systems such a costly method may introduce noticeable losses, or occupy too large volume. In this work, a load-pull technique to study these interactions after the RF power amplifiers (PA), where RF performance will be affected with respect to distortion, signal quality and efficiency, is proposed.

The interaction between the signals transmitted by different PAs can be illustrated as follows: At any given time instance the amplitude and phase of the output signal from a PA and the coupled signal to that PA, will vary with respect to each other, resulting in a changing reflection coefficient as function of time. We propose a technique to emulate this time variant reflection coefficient in a measurement setup by using representative modulated signals, both at the input of the device-undertest (DUT), as well as at the output of the DUT. Active load pull is performed at the output, where the goal is to recreate the coupling between PAs. The difference between the technique proposed and conventional load pull [2] is the constant reflection coefficient associated with the latter, whereas the former presents a controlled time-variant reflection coefficient to the DUT.

Earlier we partly demonstrated such a method, which we verified by using two PAs [3]. However, in that work, the inherent distortion from the PAs was not compensated for, and thus effects were not uniquely determined by coupling. Furthermore, the signals on the PAs were assumed to be identical, which is not a realistic condition in a real system. Moreover, delay inherent to the measurement system, was not explicitly neutralized. In this work these effects are accounted for, firstly by applying digital pre-distortion to the transmitting signal, which effectively isolates the effects caused by coupling. Secondly, the signals used are more realistic by demonstrating two cases with different cross-correlation. Finally, system delay is eliminated in the calibration procedure.

\section{THEORY}

PA interaction can be split into reflection and coupling, see Fig. 1. The N-port S-parameter block contains all coupling and reflective effects after the PAs, independent of their origin. In an ideal array, the isolation is perfect $\left(S_{i, n}=0, i \neq n\right)$, and the PAs are well matched $\left(S_{i i}=0\right)$, where $i$ and $n$ are the element indices. Thus at the output of a given PA the incoming wave $a_{2}^{(i)}=0$. However, in practice, without these conditions, $a_{2}^{(i)}$ will be non-zero. These effects will be shown to be dependent on the signals, the linear coupling and matching given by the Sparameters $S_{i, n}$.

The resulting $a_{2}^{(1)}$ at the first PA is given by:

$$
a_{2}^{(1)}=S_{11} b_{2}^{(1)}+S_{12} b_{2}^{(2)}+\ldots+S_{1 N} b_{2}^{(N)} .
$$

The time-varying $b_{2}^{(i)}$ can be measured. The S-parameters are known from simulation or an antenna measurement, and can be assumed to be time-invariant. $a_{2}^{(i)}$ can be calculated using equation (1), and the proposed time-variant load pull can be performed in an iterative fashion, where the presented reflection coefficients are given by:

$$
\Gamma_{\text {load }}^{(i)}=\frac{a_{2}^{(i)}}{b_{2}^{(i)}} .
$$

Typically the reflected component is smaller than the coupled component. Thus for a well-matched system with negligible reflected component, equation (1) simplifies to:

$$
a_{2}^{(1)}=S_{12} b_{2}^{(2)}+\ldots+S_{1 N} b_{2}^{(N)} .
$$

This simplification will be validated by the experiments. 


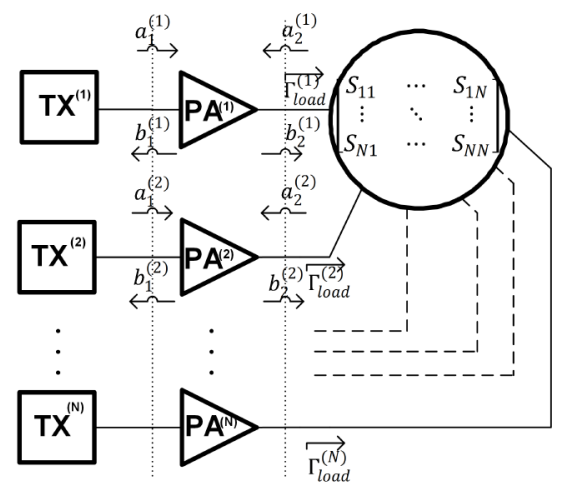

Fig. 1. $\mathrm{N}$ interacting PAs, the coupling is given by N-port S-parameters.

\section{A. Emulation theory}

To transfer the described method to a practical measurement setup, it is unfeasible to include all PAs. Therefore, a number of DUTs is used as representatives for each type of PA in the array. If all array elements are identical, a single DUT can be representative for the entire array. Where normally all PAs are switched on simultaneously, the proposed technique translates this in a time-division manner, where at a certain time slot, the DUT represents a specific PA, in the next time slot, the DUT represents the next element. Specifically, in each time slot, the DUT is driven with the corresponding input signal $a_{1}^{(i)}$, of the $i^{\text {th }}$ PA. Simultaneously, the output of the DUT is load-pulled with the corresponding known $a_{2}^{(i)}$ as defined in (3). This is performed in an iterative fashion as illustrated in Table I. It can be seen that at the initialization not all $b_{2} \mathrm{~s}$ are known, thus the calculation of $a_{2} \mathrm{~s}$ is first incomplete, however during the measurement procedure, after some iterations, more information become available, and the coupling effect can be represented.

\section{MEASUREMENT SYSTEM}

A measurement setup was constructed which enables both emulation load pull measurements, as well as verification measurements. A schematic overview is given in Fig. 2. The emulation setup consists of two transmitters (TX1 and TX2) as well as two receivers (RX1 and RX2). Each combination of RX and TX consists of a National Instruments vector signal transceiver with up to $200 \mathrm{MHz}$ analog bandwidth. To ensure coherent generation and acquisition the transmitters share a single local oscillator (LO) and the receivers share another LO. The LOs as well as sampling clocks are locked to a single reference. Two trigger signals control the start of generation and acquisition. TX1 generates the input signal to the DUT, whereas TX2 generates the emulated $a_{2}$ wave. A driver, protected by an isolator, amplifies the signal to the required amplitude. The $a_{2}$ and $b_{2}$ waves are measured by the RXs, using a directional coupler. Given the case of array with two elements, the verification setup is similar to the emulation setup, where the driver and the isolator are replaced by a second PA, DUT2, which is identical to DUT1. Coupling level can be controlled by inserting any
TABLE I

ILLUSTRATION OF EMULATION ITERATIVE PROCEDURE: N ANTENNAS, N DIFFERENT SIGNALS, L ITERATIONS

\begin{tabular}{|c|c|c|c|}
\hline \multirow[t]{2}{*}{ Steps } & \multicolumn{2}{|r|}{ Transmitting } & \multirow{2}{*}{$\begin{array}{c}\text { Receiving } \\
\mathrm{R} \times 1\end{array}$} \\
\hline & $T x 1$ & $T \times 2$ & \\
\hline \multirow[t]{5}{*}{1} & $a_{1}^{(1)}$ & - & $b_{2}^{(1,1)}$ \\
\hline & $a_{1}^{(2)}$ & $S_{2,1} \cdot b_{2}^{(1,1)}$ & $b_{2}^{(2,1)}$ \\
\hline & $a_{1}^{(3)}$ & $S_{3,1} \cdot b_{2}^{(1,1)}+S_{3,2} \cdot b_{2}^{(2,1)}$ & $b_{2}^{(3,1)}$ \\
\hline & & $\ldots$ & $\ldots$ \\
\hline & $a_{1}^{(N)}$ & $S_{N, 1} \cdot b_{2}^{(1,1)}+S_{N, 2} \cdot b_{2}^{(2,1)}+\ldots+S_{N,(N-1)} \cdot b_{2}^{(N-1,1)}$ & $b_{2}^{(N, 1)}$ \\
\hline \multirow[t]{4}{*}{2} & $a_{1}^{(1)}$ & $S_{1,2} \cdot b_{2}^{(2,1)}+. .+S_{1, N} \cdot b_{2}^{(N, 1)}$ & $b_{2}^{(1,2)}$ \\
\hline & $a_{1}^{(2)}$ & $S_{2,1} \cdot b_{2}^{(1,2)}+. .+S_{2, N} \cdot b_{2}^{(N, 1)}$ & $b_{2}^{(2,2)}$ \\
\hline & $\ldots$ & $\ldots$ & $\ldots$ \\
\hline & $a_{1}^{(N)}$ & $S_{N, 1} \cdot b_{2}^{(1,2)}+S_{N, 2} \cdot b_{2}^{(2,2)}+. .+S_{N,(N-1)} \cdot b_{2}^{(N-1,2)}$ & $b_{2}^{(N, 2)}$ \\
\hline$\ldots$ & 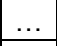 & $\ldots$ & .. \\
\hline \multirow[t]{5}{*}{$\mathrm{L}$} & $a_{1}^{(1)}$ & $S_{1,2} \cdot b_{2}^{(2, L-1)}+. .+S_{1, N} \cdot b_{2}^{(N, L-1)}$ & $b_{2}^{(1, L)}$ \\
\hline & $a_{1}^{(2)}$ & $S_{2,1} \cdot b_{2}^{(1, L)}+. .+S_{2, N} \cdot b_{2}^{(N, L-1)}$ & $b_{2}^{(2, L)}$ \\
\hline & $a_{1}^{(3)}$ & $S_{3,1} \cdot b_{2}^{(1, L)}+S_{3,2} \cdot b_{2}^{(2, L)}+. .+S_{3, N} \cdot b_{2}^{(N, L-1)}$ & $b_{2}^{(3, L)}$ \\
\hline & $\ldots$ & $\ldots$ & $\ldots$ \\
\hline & $a_{1}^{(N)}$ & $S_{N, 1} \cdot b_{2}^{(1, L)}+S_{N, 2} \cdot b_{2}^{(2, L)}+. .+S_{N,(N-1)} \cdot b_{2}^{(N-1, L)}$ & $b_{2}^{(N, L)}$ \\
\hline
\end{tabular}

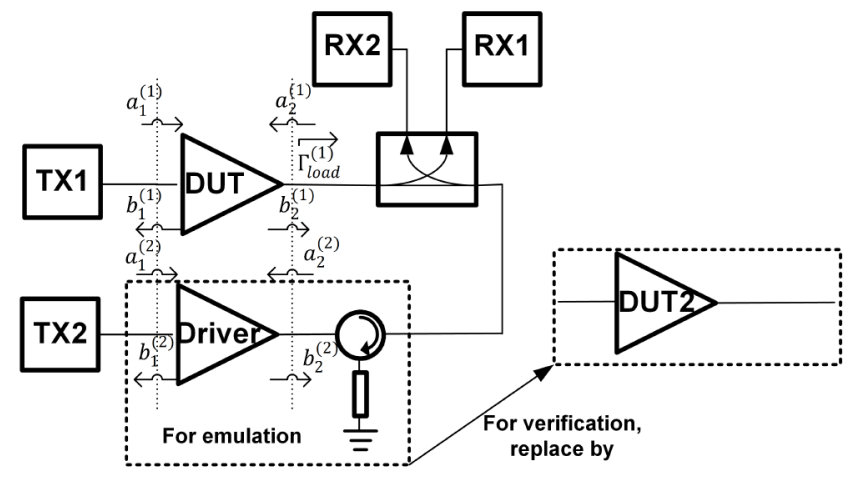

Fig. 2. Schematic representation of the emulation setup. For verification measurements the driver and isolator are replaced by DUT2.

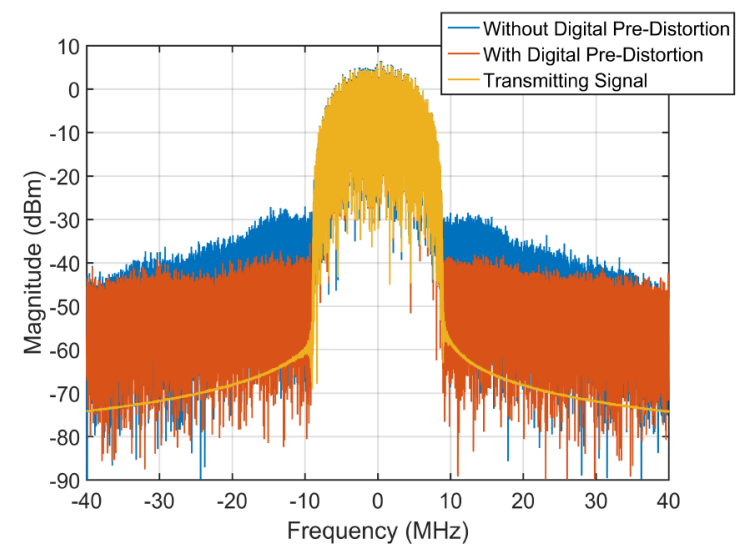

Fig. 3. Magnitude spectrum of the transmitted signal, measured received signal without DPD, and measured received signal with DPD. 


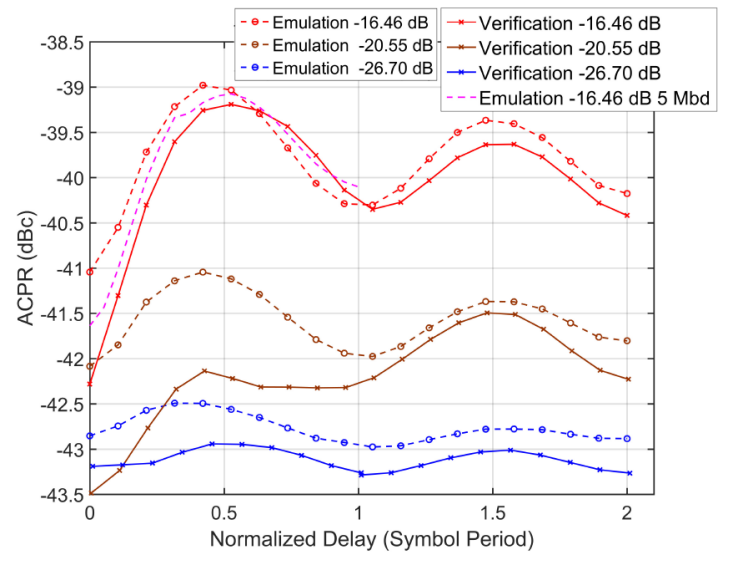

Fig. 4. Measured ACPR as a function of delay between two PAs at different coupling levels and high signals' correlation.

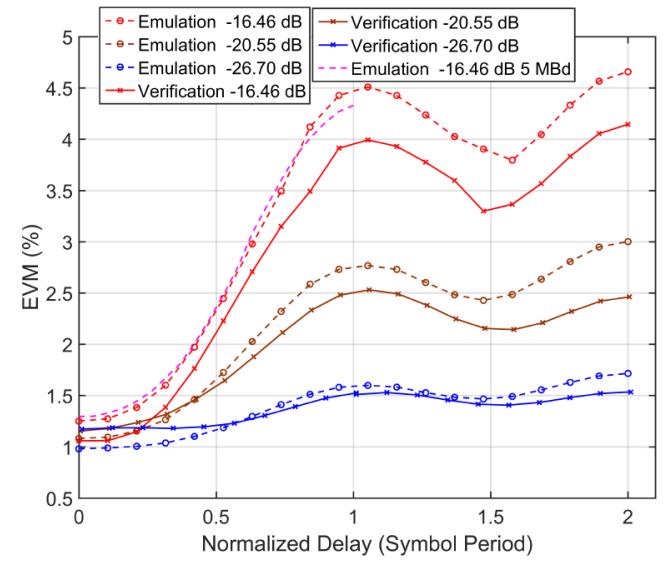

Fig. 5. Measured EVM as a function of delay between two PAs at different coupling levels and high signals' correlation.

two-port device, e.g. an attenuator, between DUT2 and the directional coupler. The measurement setup is calibrated at the reference plane at the output of DUT1, using short, open, load, and power calibration. Delays due to trigger delay, PA delay and differences in electrical path length are quantified and compensated for. The delay along the coupling path is of interest and is digitally varied in the measurement.

\section{MEASUREMENT RESULTS}

The test signal is 16-QAM, root-raised cosine pulse-shaped with roll-off factor of 0.8 . Carrying $2^{17} \mathrm{bits}$, the generated baseband signal is up-converted to $2.14 \mathrm{GHz}$. Unless otherwise noted, the test signal is transmitted at $10 \mathrm{MBd}$ or $40 \mathrm{Mbit} / \mathrm{s}$. Two application cases are considered. In the first case, the signals transmitted by the two PAs are highly correlated, representing a low usage scenario beamforming setup. Whereas in the other scenario, the signals are potentially highly uncorrelated, such as in a high usage scenario in a rich scattering environment, massive MIMO system.

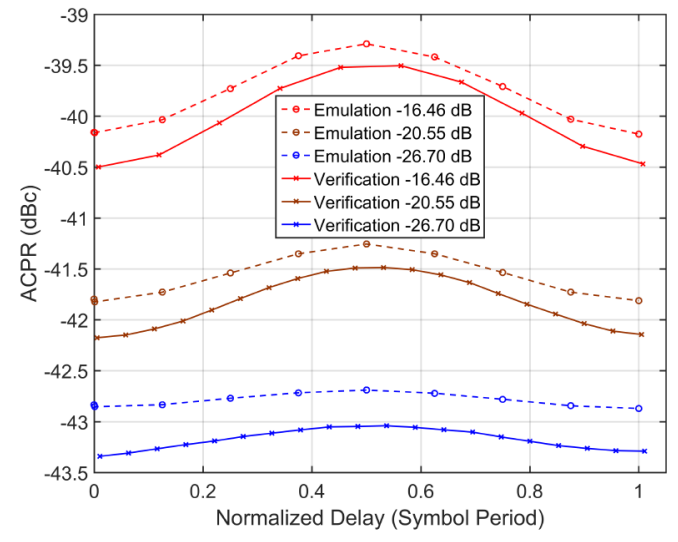

Fig. 6. Measured ACPR as a function of delay between two PAs at different coupling levels and low signals' correlation.

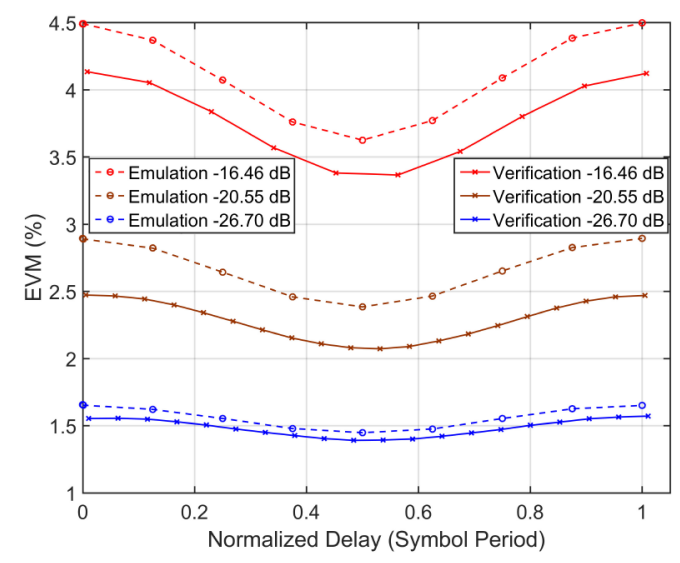

Fig. 7. Measured EVM as a function of delay between two PAs at different coupling levels and low signals' correlation.

\section{A. Digital Pre-distortion}

In order to study the coupling effects, the PAs inherent nonlinear characteristics were compensated for, by performing digital pre-distortion (DPD) at the input of the devices. The method used to identify the parameters of the pre-distorter is a variant of the indirect learning architecture [4], as described in [5]. Driving the DUT, terminated with $50 \mathrm{Ohm}$, with the test signal results in nonlinear distortion at the output, which can be quantified as normalized mean-square error of $-27.9 \mathrm{~dB}$, and adjacent channel power ratio (ACPR) of $-35.3 \mathrm{dBc}$. After implementing DPD, these values were reduced to $-37.2 \mathrm{~dB}$, and -43.8 $\mathrm{dBc}$, respectively. This improvement is also shown in Fig. 3, where the magnitude spectrum of the transmitting, and receiving signal with and without DPD are shown. This implementation effectively allows lower coupling levels to be studied, while ensuring the distortion is influenced only through the coupling effects. 


\section{B. Experimental results}

A two element array with different signals, was emulated and verified. ACPR, and error-vector magnitude (EVM) as function of coupling factor and delay are measured on a $6 \mathrm{~W}$ CREE CGH40006S GaN HEMT PA. Fig. 4 and 5 present the case of highly correlated signals, while Fig. 6 and 7 present the low correlation case. The differences between the two cases can only be attributed to the different time-variant impedances the DUT sees. The coupling factors used represent typical values for low $\mathrm{GHz}$ patch antennas arrays. The delays were chosen to exceed typical expected values to represent higher bandwidth systems as well. It can be observed that both coupling level and delay affect system performance.

To generalize the results, the delay between the two elements are normalized to the symbol period, validated by the agreement between 5 and $10 \mathrm{MBd}$ results at $-16.46 \mathrm{~dB}$ coupling in Fig. 4. The delay dependency demonstrates the importance of delay for future systems with increased bandwidth.

The results demonstrate good agreement between emulation and verification, which validates the simplification of (3). As for output power and efficiency, the average discrepancies between emulation and verification were $0.3 \mathrm{~dB}$ and $1.6 \%$, respectively.

\section{CONCLUSION}

A time-varying load pull technique has been proposed. It was demonstrated through experiment that such technique is suitable for emulation of antenna array coupling effects on system performance without the need for array construction.

\section{ACKNOWLEDGMENT}

The results in this paper are partly the result of the project MET5G - Metrology for 5G communications. This project has received funding from the EMPIR programme co-financed by the Participating States and from the European Union's Horizon 2020 research and innovation programme. The authors would like to thank Katharina Hausmair and Prof. Thomas Eriksson, both at the Chalmers University of Technology, for fruitful discussions on digital pre-distortion.

\section{REFERENCES}

[1] F. Boccardi, R. W. Heath, A. Lozano, T. L. Marzetta and P. Popovski, "Five disruptive technology directions for 5G," in IEEE Communications Magazine, vol. 52, no. 2, pp. 74-80, February 2014.

[2] M. Marchetti, M. J. Pelk, K. Buisman, W. Neo, M. Spirito, and L. C. N. de Vreede, "Active harmonic load-pull with realistic wideband communications signals," IEEE Trans. Microw. Theory Tech., vol. 56, no. 12, pp. 2979-2988, Dec. 2008.

[3] D. Nopchinda, and K. Buisman, "Emulation of array coupling influence on RF power amplifiers in a measurement setup," in Microwave Measurement Conference (ARFTG), San Francisco, 2016, pp. 1-4.

[4] C. Eun, and E. Powers, "A new Volterra predistorter based on the indirect learning architecture," IEEE Trans. Signal Process., vol. 45, no. 1, pp. 223-227, Jan. 1997.

[5] J. Chani-Cahuana, C. Fager, and T. Eriksson, "A new variant of the indirect learning architecture for the linearization of power amplifiers," in $E u$ ropean Microwave Conference (EUMC), Paris, 2015, pp. 1295-1298. 